\title{
An Electrical-Analog Method for Transient Heat-Flow Analysis
}

\section{A. F. Robertson and Daniel Gross}

\begin{abstract}
An electronic instrument is described which permits solution of transient heat-flow problems by use of direct analogy to electrical networks. The instrument is of the so-called "fast-time" type, simulating the transient involved in times of the order of $10^{-6}$ that of the thermal prototype. A photoformer type of input-signal generation is used and this, together with a variable-frequency master oscillator, permits flexibility of input-signal waveform and time scale.

Some applications of the instrument to the field of fire research are described. The discussion of errors includes consideration of those resulting from the lumping of circuit elements.
\end{abstract}

\section{Introduction}

Laboratories in which studies are performed on the fire-resistance behavior of building structural components have been frequently asked to predict or estimate the fire performance of structures that have not been tested. In some cases data are available upon which useful estimates may be based. In most cases, however, the quality of the resulting estimates is largely dependent on the experience and feeling the estimator has for the problem. In an effort to assist in placing such estimates on a more exact basis, an electronic device for performing the necessary computations was designed and constructed at the Bureau.

Fire-resistance tests are performed on portions of buildings for the purpose of determining the suitability of the construction in withstanding the effects of fire. While mechanical behavior may frequently limit the usefulness of the structure in this respect, it often happens that thermal transmission is the critical factor in determining its ability to resist fire. The procedure used during such tests [1] ${ }^{1}$ requires application to the furnace-enclosed structure of a time-varying temperature function corresponding to the standard fire exposure. The procedure also permits heat losses to occur from the unexposed portion of the specimen by both radiation and convection. These conditions make it impractical to use analytical methods for solution of the heat-transfer equation.

Because of this it seemed desirable to use some high-speed approximation methods for computing the thermal behavior of structures exposed to fire. Both digital and conventional analog computers were considered and used with a certain degree of success for approximate solutions of such problems. However, it seemed likely that use of the direct analogy between thermal and electrical circuits might permit greater flexibility in the solution of problems and simplify the "coding".

The device constructed is somewhat similar to the one developed by Lawson \& McGuire [2]. This makes use of the analogy between electrical and thermal circuits directly without the need for a large assembly of electronic mathematical-operator units

\footnotetext{
1 Figures in brackets indicate the literature references at the end of this paper.
}

which, in conventional computers, are assembled in appropriate fashion to solve the mathematically expressed relationship describing the heat-transfer problem. This direct use of the analogy permits building a basic signal-generating and measuring unit which is much more compact than the usual analog computer. The theory, construction problems, operation, and limitations of this device are briefly described.

\section{Analogies}

In many engineering investigations of the physical behavior of mechanical, electrical, fluid dynamic, thermal, and optical systems, the complexity of analytical or approximate numerical solutions of the mathematical formulation of the problem may become very difficult and excessively time consuming. In the past this has often resulted in the use of intuitive design procedures. In such cases an approximate prototype of the system involved was constructed and experimental studies made of its performance. In many instances it was difficult to vary the design parameters without extensive rebuilding of the prototype. Because of this, scientists and engineers concerned with the design of such systems have, with increasing frequency, made use of analogies. Thus an electrical system may be analogous to a mechanical, thermal, or other system. provided there is a likeness between the two systems. Usually such a likeness is not one of appearance or form but a resemblance of physical behavior. Thus, if it can be shown that the mathematical descriptions of the behavior of two different systems are similar, then it is usually possible to use one system as a means for studying the behavior of the other. The ease with which electrical circuits can be assembled and their behavior studied have made them particularly useful as analogs.

Analogs have long been used by men, maps perhaps represent one of the earliest useful forms. Kirchhoff [3] in 1845 utilized the analogy between current flow in a plate and flux distribution between charged parallel-line conductors in a uniform medium to determine the capacitance between certain configurations of such conductors. Since then, hundreds of publications have appeared on the subject of analog 
techniques. Higgins [4] has compiled a very extensive bibliography of electroanalogic methods in which appear references to almost a thousand original papers. Soroka's paper [5] provides one of the better introductions to the great versatility of analog techniques in the solution of engineering problems. His later book [6] provides a detailed exposition of many possible analog methods and techniques.

Beuken [7] presented one of the early descriptions of the use of electrical circuits for analysis of transient heat-flow phenomena. Paschkis [8] has constructed and made extensive use of a similar but larger device. Both of these instruments are of the so-called "longtime" type. This implies that the thermal transients are studied by means of electrical circuits requiring transient periods of several minutes or more duration. This permits the use of potentiometric-type instruments for data-recording purposes. The major drawback to the use of such systems is the requirement for use of rather large capacitors.

Lawson and McGuire [2] describe a device of the "fast-time" type. Here the electrical transient is completed in a few milliseconds and the problem repetitively solved, measurements being made by means of cathode-ray presentation of the data. This latter device was intended primarily for solution of problems quite similar to those considered here. In their paper they present a rather comprehensive exposition of the basis of the electrical-thermal transient analogy, together with a discussion of means of application to one-dimensional space problems and some discussion of the possible errors involved in the use of lumped circuit elements. Because of the extensive discussions in the literature of the various types of analog systems possible it would serve little purpose to repeat the descriptions here. A brief indication will, however, be made of the theoretical basis for use of electrical models for solution of transient heat-flow problems. This should not be taken to imply that heat-flow problems are the only type for which the instrument to be described may be used. It consists essentially of a voltage versus time signal-generation unit, means for application of the generated signal repetitively to an electrical network, and provision for measuring the transient voltages which are developed within the model. Thus, any electrical network that can be used as an analogy of another type of system may be used with this instrument.

By definition the rate of heat flow $\partial Q / \partial t$ in the direction of a one-dimensional thermal gradient is given as the product of the conductivity $k$, the area $A$ across which heat flow is taking place, and the temperature gradient $\partial \theta / \partial x$ or,

$$
\partial Q / \partial t=-k A \partial \theta / \partial x,
$$

the negative sign indicating that flow takes place in the direction of a negative temperature gradient.

The difference in the heat-flow rates into and out of a small elemental section along the path of heat flow, or the heat-flow gradient, is equal to the product of the volumetric heat capacity $\rho s$, rate of temperature rise $\partial \theta / \partial t$, and area over which heat is flowing.
Thus

$$
\frac{\partial}{\partial x} \frac{\partial Q}{\partial t}=-A \rho s \frac{\partial \theta}{\partial t}
$$

Combining these two equations we obtain,

$$
\frac{\partial \theta}{\partial t}=\frac{k}{\rho s} \frac{\partial^{2} \theta}{\partial x^{2}} .
$$

This is the one-dimensional form of the well-known Fourier equation of heat conduction.

In a similar manner we may develop the equations for a series-resistance, shunt-capacitance electrical transmission line for the particular case in which the capacitance is assumed proportional to the volume of the line. ${ }^{2}$ From the definition of electrical units and Kirchoff's laws,

$$
i=-\frac{a}{r} \frac{\partial v}{\partial x}
$$

where $i$ is the current, $r$ the resistivity of the line material, $a$ the cross-sectional area and $\partial v / \partial x$ the voltage gradient. The current gradient in the direction of flow becomes,

$$
\frac{\partial i}{\partial x}=-c a \frac{\partial v}{\partial t}
$$

where $c$ is the capacitance per unit volume and $\partial v / \partial t$ is the rate of voltage rise. Equations (4) and (5) may be combined to form,

$$
\frac{\partial v}{\partial t}=\frac{1}{r c} \frac{\partial^{2} v}{\partial x^{2}}
$$

Equations (3) and (6) are obviously similar and thus justify the use of the electrical system as an analog to the thermal system. In a similar way, eq (1) and (4) and (2) and (5) may be considered as analogous. Table 1 has been prepared to list the properties of the two systems in analogous form, assuming equivalence of the cross-sectional areas of the thermal and electrical circuits.

Because of difficulties of constructing within small dimensions, electrical models of such transmission lines in which the capacitance is distributed along its length, it is common practice to approximate this by a lumped system network. Three such networks, incorporating a series of either $\mathbf{T}, \mathbf{L}$, or $\mathbf{P i}$ sections, such as those bounded by dotted brackets are shown in figure 1. It may be seen that although the three lines consist of different arrangements of resistance and capacitance elements, the lines are identical except for portions of the first and last sections. The resistors, $R$, of these lines then correspond to the total resistance of the thermal circuit divided by the number of sections, $N$, used in the electrical model. In a similar way the capacitors correspond to the sectional heat capacity of

2 This differs from the geometrical considerations commonly used in determining capacitance. Such treatment is used, however, to permit development of the equations shown and to thus maintain true similitude of the thermal-electrical the equation
analogy. 
TABLE 1. Analogous elements of thermal and electrical systems

\begin{tabular}{|c|c|c|c|c|c|}
\hline \multicolumn{3}{|c|}{ Thermal } & \multicolumn{3}{|c|}{ Electrical } \\
\hline Property & Symbol & Unit & Property & Symbol & Unit \\
\hline $\begin{array}{l}\text { Temperature } \\
\text { Time } \\
\text { Heat-flow rate } \\
\text { Heat capacity } \\
\text { Conductivity } \\
\text { Length } \\
\text { Temperature gradient } \\
\text { Rate of temperature rise }\end{array}$ & $\begin{array}{c}\theta \\
t_{t} \\
\partial Q / \partial t_{t} \\
\rho s \\
k \\
x_{t} \\
\partial \theta / \partial x_{t} \\
\partial \theta / \partial t_{t}\end{array}$ & $\begin{array}{c}\circ \mathrm{C} \\
\text { second } \\
\text { watt } \\
\text { joule } / \mathrm{cm}^{3} \circ \mathrm{C} \\
\text { watt } / \mathrm{cm}^{\circ} \mathrm{C} \\
\mathrm{cm} \\
\circ \mathrm{C} / \mathrm{cm} \\
\circ \mathrm{C} / \text { second }\end{array}$ & $\begin{array}{l}\text { Voltage } \\
\text { Time } \\
\text { Current } \\
\text { Capacitance } \\
\text { Conductivity. } \\
\text { Length } \\
\text { Voltage gradient } \\
\text { Rate of voltage rise }\end{array}$ & $\begin{array}{c}v \\
t_{e} \\
i \\
c \\
1 / r \\
x_{e} \\
\partial v / \partial x_{e} \\
\partial \theta / \partial t_{e}\end{array}$ & $\begin{array}{c}\text { volt } \\
\text { second } \\
\text { microampere } \\
\text { microfarad } / \mathrm{cm}^{3} \\
\text { 1/megohm cm } \\
\mathrm{cm} \\
\text { volt } / \mathrm{cm} \\
\text { volt } / \text { second }\end{array}$ \\
\hline
\end{tabular}
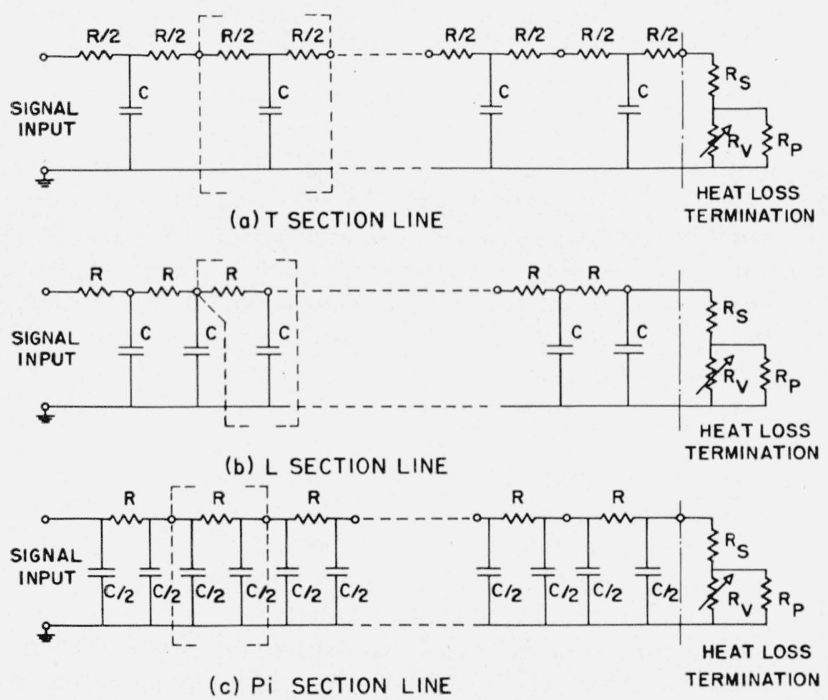

Figure 1. Comparison of $\mathbf{T}, \mathbf{L}$, and $\mathbf{P i}$ section lines.

the thermal model, $\rho s x / N$. The termination, $R_{f}$, comprising a varistor, or positive voltage coefficient resistor $R_{v}$, together with series and parallel resistors, $R_{s}$ and $R_{p}$, if necessary, is used to simulate the convective and radiative heat losses from the cool end of the thermal structure. The detailed technique for using such elements was described by Lawson and McGuire [2].

Again considering the similarity between eq (3) and (6) together with the analogous properties shown in table 1 , it is common practice to substitute electrical for thermal properties directly and to solve the electrical problem, but to think of the solution as a thermal one. In performing this conversion it will be found convenient to use scaling factors. Thus rather than substituting directly electrical capacitance for heat capacity we have,

$$
c=\frac{\rho s}{n} \quad \text { or } n=\frac{\rho s}{c},
$$

similarly for resistivity

$$
r=\frac{1}{m k} \quad \text { or } m=\frac{1}{k r},
$$

and for time

$$
t_{e}=\frac{t_{t}}{m n \phi} \quad \phi=\frac{t_{t}}{m n t_{e}}
$$

where $n, m$, and $\phi$ are the capacity, resistivity, and time-scaling factors, respectively. Factors such as these may be used both to obtain control over the size of the electrical components used as well as to provide a legitimate manner for conversion from thermal to electrical units as implied by these equations. This latter objective may, however, also be obtained by the assumption of such conversion ratios without their explicit mention. Table 2 shows the modifications of the analogous thermal and electrical elements when capacity, resistivity, and time-scaling factors are employed. The relationships developed maintain the identity of eq (4) and (5).

TABLE 2. Modification of analogy relationships by application of scaling factors

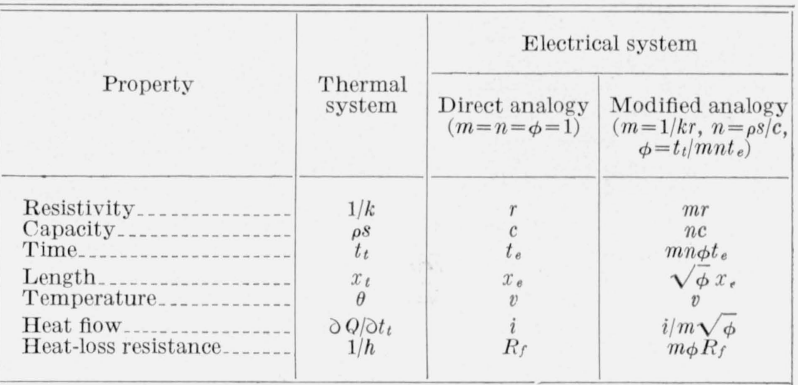

As an example of the manner in which an electrical model may be constructed it will be assumed that we wish to represent a concrete floor slab which is $10.16 \mathrm{~cm}$ (4 in.) thick. We will assume that the model is to have 20 sections of the $\mathbf{L}$ type. This problem is shown in figure 2.

For a resistivity scaling factor of $m$ we have for the resistance,

$$
\frac{x}{k N}=\frac{m r x}{N}=m R, \quad \text { or } R=\frac{x}{N m k} .
$$

Inserting numerical values and using a consistent set of dimensions for both electrical and thermal systems,

$$
R=\frac{x}{N m k}=\frac{10.16}{(20)(0.0156)(m)}=\frac{32.6}{m} .
$$

If $m=652$, each network resistor is $0.050 \mathrm{meg}$, a convenient value. 


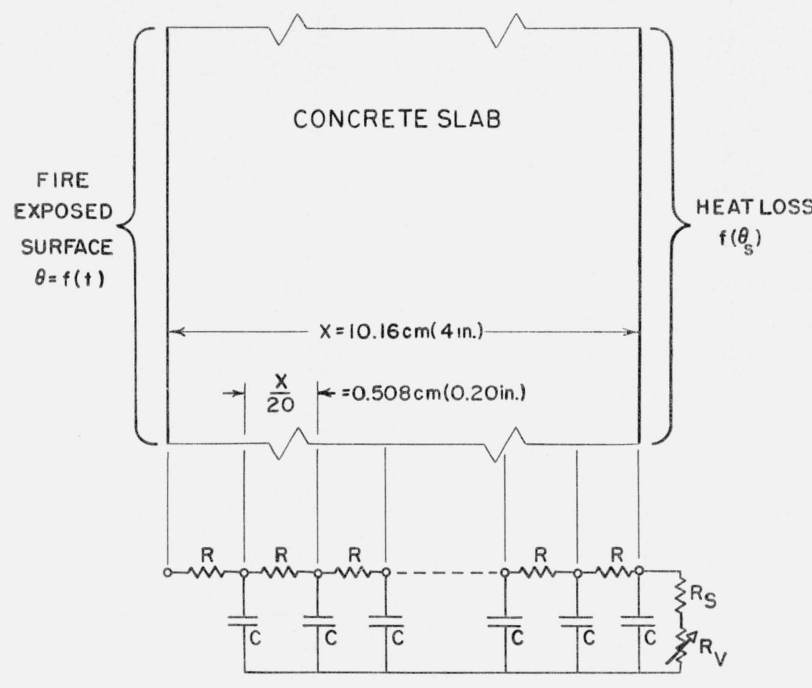

ELECTRICAL MODEL (L. SECTION)

Figure 2. Method of development of electrical model for a fireexposed concrete slab.

Thermal properties: $k=0.0156 \mathrm{w} / \mathrm{cm}^{\circ} \mathrm{C}\left(0.90 \mathrm{Btu} / \mathrm{hr} \mathrm{ft}{ }^{\circ} \mathrm{F}\right) ; \rho=2.24 \mathrm{~g} / \mathrm{cm}$ $\left(140 \mathrm{lb} / \mathrm{ft}^{3}\right) ; s=0.837$ joule $/ \mathrm{g} \circ \mathrm{C}\left(0.2 \mathrm{Btu} / \mathrm{lb}{ }^{\circ} \mathrm{F}\right)$. Electrical values: $R=50 \mathrm{~K}$ $R_{s}=400 \mathrm{~K} ; R_{\mathrm{v}}=$ varistor; $C=449 \mu \mu \mathrm{f}$.

In a similar fashion, for a capacity scaling factor of $n$ we have,

$$
\frac{\rho s x}{N}=\frac{n c x}{N}=n C \quad \text { or } C=\frac{\rho s x}{N n} .
$$

Again inserting numerical values,

$$
C=\frac{\rho s x}{N n}=\frac{(2.24)(0.837)(10.16)}{(20)(n)}=\frac{0.953}{n} .
$$

If $n=2120$, each network capacitor is $0.000449 \mu \mathrm{f}$.

Finally, for $\phi=1$,

$$
t_{l}=m n t_{e}=(652)(2120) t_{e}=1.38 \times 10^{6} t_{e} .
$$

Thus, in using the scale ratios proposed, the thermal time scale has been compressed by a factor of about $10^{6}$. To provide for a thermal period of $8 \mathrm{hr}$, we represent this by $8 \times 10=80$ divisions of $6 \mathrm{~min}$ each. Each division therefore corresponds to $6 \times 60 / 1.38 \times 10^{6}=261 \mu \mathrm{sec}$, and the master oscillator is adjusted to operate at a frequency of $3.83 \mathrm{kc}$. The appropriate varistor assembly is connected at the end of the network corresponding to the upper surface of the floor slab to represent radiative and convective heat losses.

If, as is often likely, it is found that capacitors or resistors of the particular sizes suggested are not readily available the value of $m$ and/or $n$ may be changed and the frequency similarly adjusted. The ease with which this may be done is one of the desirable features of this particular instrument. It not only greatly facilitates construction of models but, as shown in table 2, permits the use of a given model to simulate a whole range of structure sizes simplyfby independent change of the time scale, i. e., a change in $\phi$. When this is done it is important to remember that all dimensions of the thermal system are changed. Thus in the analog of a multicomponent thermal body a change of time scale will result in the simulation of a new thermal system in which all dimensions in the direction of heat flow are scaled in the same ratio. If special terminations have been used to simulate heat-loss conditions for such a system, care must be taken to adjust these separately according to the scaling relationships shown in table 2.

\section{General Description of Instrument}

The instrument constructed was of the "fasttime" type in which a particular problem is solved repetitively at a speed much higher than that used for the thermal prototype. The ratio of the speed of the electrical operation to the speed of the thermal process was of the order of $10^{6}$. This requires cathode-ray presentation for read-out of the repetitively solved problem. In order to reduce the cost and time involved in its construction, commercial electronic instruments were used wherever it seemed practical. $^{3}$

A block diagram of the device is shown in figure 3 . This has been divided into three interrelated functional units: (a) The signal generator; (b) the electrical model; and (c) the measuring circuits. The three power supplies are not shown.

The signal generator unit selected was of the photoformer type $[9,10]$. This type was chosen because of the great flexibility of waveforms possible with such a system and the ease with which they could be interchanged. A cathode-ray tube is arranged with an opaque mask covering a portion of its face. A phototube with appropriate amplifier is arranged to view the face of the cathode-ray tube. The amplified output of the phototube is applied to the vertical deflection plates of the cathode-ray tube in such a way that when the spot is "seen" by the phototube it is deflected downwards and forced to be partially hidden by the mask. The application of a relaxation-type timing sweep to the horizontal plates of the cathode-ray tube results in the spot following the mask outline on the cathode-ray tube screen. Since for given operating conditions the position of the spot on the screen bears a fixed relationship to the voltage applied to the deflection plates, a voltage-time signal may be taken from them which is controlled by the shape of the mask.

In practice, a commercial oscilloscope was used in conjunction with a photomultiplier tube as the generator. A special impedance converter was built to modify the balanced high-impedance signal applied to the deflection plates into a low-impedance single-ended output signal suitable for application to the model.

The electrical model was custom-made for each thermal situation being simulated. Figure 4 shows a photograph of one of the models used. It consists

3 The special components and instruments used in the assembly are listed in the appendix of this paper. 


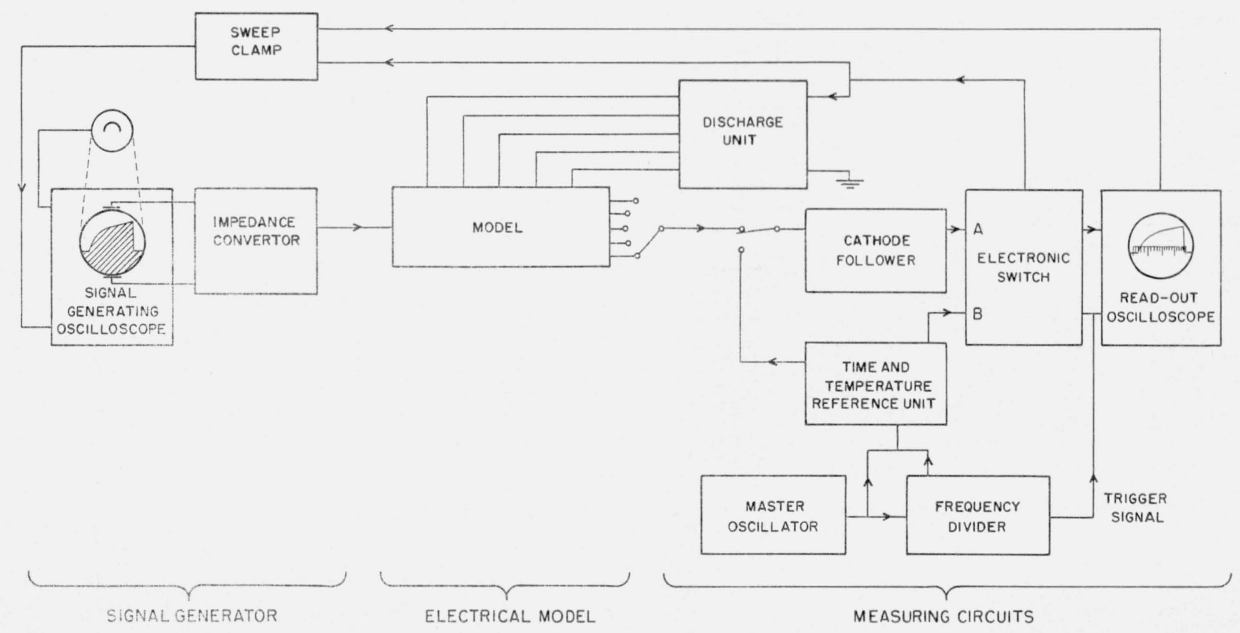

Figure 3. Block diagram of the instrument.

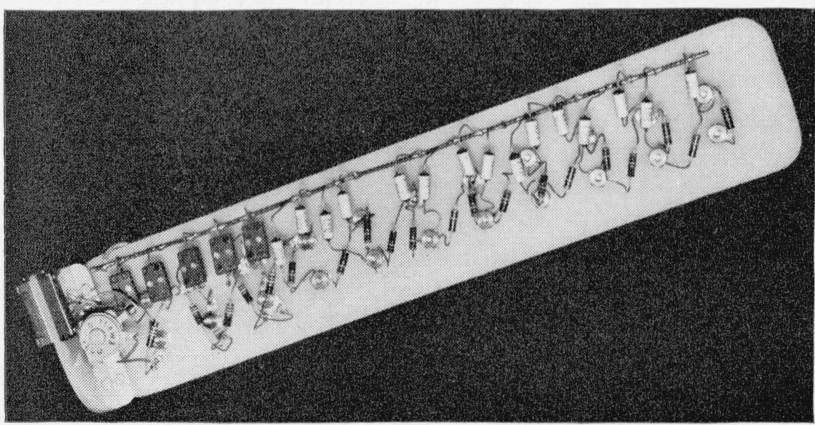

Figure 4. Tiew of an electrical model.

of a series of resistors representing the thermal resistance of the prototype and a group of capacitors shunting these resistors to ground and representing the heat capacity of the thermal system. The assembled model assumes the form of a plug-in unit, provisions being made for twenty discharge points.

The measuring unit comprised a number of circuits which may be briefly described as follows:

1. The master oscillator is a variable-frequency square-wave generator used as a time-reference source.

2. The frequency-divider circuit generates the decade-time markers and submultiples of these for synchronizing signals.

3. The temperature and time-reference unit is a mixing device which assembles the calibrated time scale with a variable d-c voltage for making "temperature" measurements.

4. The discharge unit is a special circuit used for discharging the capacitors used in the model. This discharge process is accomplished every alternate sweep of the viewing oscilloscope during the period of presentation of the time and temperature reference signal.

5. The cathode follower is a special high inputimpedance circuit which permits making voltage measurements on the model without serious electrical loading of it.
6. The electronic switch provides a means for alternate presentation of the signal from the model and that from the time and temperature reference unit.

7. The read-out oscilloscope provides a means for study of the electrical behavior of the model and permits direct measurement of voltage corresponding to temperature changes within the model. This oscilloscope is used to generate the time-sweep signal for the signal generator. Trigger operation is used and the sweep signal applied to the signal generator is clamped on alternate cycles in such a way that a signal is generated only when required for application to the model.

Figure 5 shows the assembled device. The power supplies have been mounted below the desk while the remainder of the equipment is assembled in two relay racks. The electrical model is seen near the midheight of the right-hand rack.

\section{Special Electronic Circuits}

Figure 6 presents the complete circuit diagram for the specially constructed portions of the unit. Also shown diagrammatically are the commercially available electronic units which formed a part of the complete device. The high- and low-voltage power supplies were of the regulated type and are not indicated in this diagram. The special considerations which were involved in the selection of the instruments and circuits follow.

\subsection{Signal-Generating Oscilloscope}

The particular oscilloscope used was of the lowaccelerating voltage, single-anode, electrostaticdeflecting type. It was chosen to permit achievement of high linearity between mask size and output signal. It was fitted with a tube having a short persistence type $\mathrm{P}-15$ phosphor. The short phosphorescent decay time, down 90 percent in $2.8 \mu$ sec, of this phosphor was found necessary to permit close following of transient mask shapes. The 


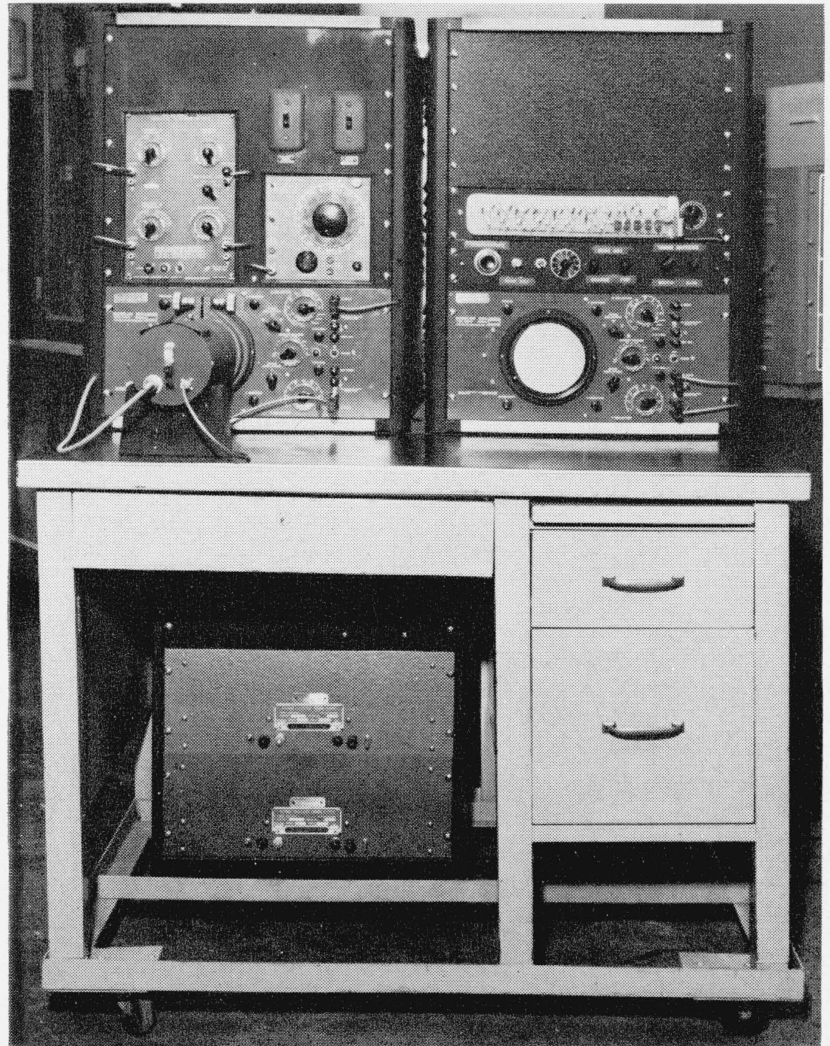

Figure 5. Transient heat-flow analyzer. photomultiplier tube associated with the oscilloscope was selected to have a spectral sensitivity which permitted efficient use with the cathode-ray tube phosphor. The high-output sensitivity of this tube was also a factor involved in its selection. A darkened hood was of course used to shield the photomultiplier from room illumination. In using this photoformer circuit it was found that the delay time of the oscilloscope direct-coupled amplifiers caused considerable hunting. This had the effect of superimposing a small a-c signal in excess of $80 \mathrm{kc}$ on the signal being generated. The presence of this highfrequency signal was not considered objectional for normal use in solving problems since it was completely filtered out by the first section of the network to which it was applied. It could be greatly reduced in amplitude by shunting the photomultiplier output by a $150 \mu \mu$ capacitor. A more proper way of eliminating this hunting would involve design of a special amplifier having short time delay and possibly incorporation of negative feedback. In using a signal generator of this type it is essential that the spot remain on the tube screen during periods when signals are not being generated. To permit this type of operation, the generating oscilloscope was modified by insertion of clamp circuits on the horizontal deflection circuit to prevent the spot from leaving the right-hand edge of the screen. The circuit used is shown in figure 7 . It does not abruptly limit spot deflection at one position but has the effect of greatly reducing the deflection sensitivity when the spot approaches the edge of the screen. This type of operation was achieved easily and

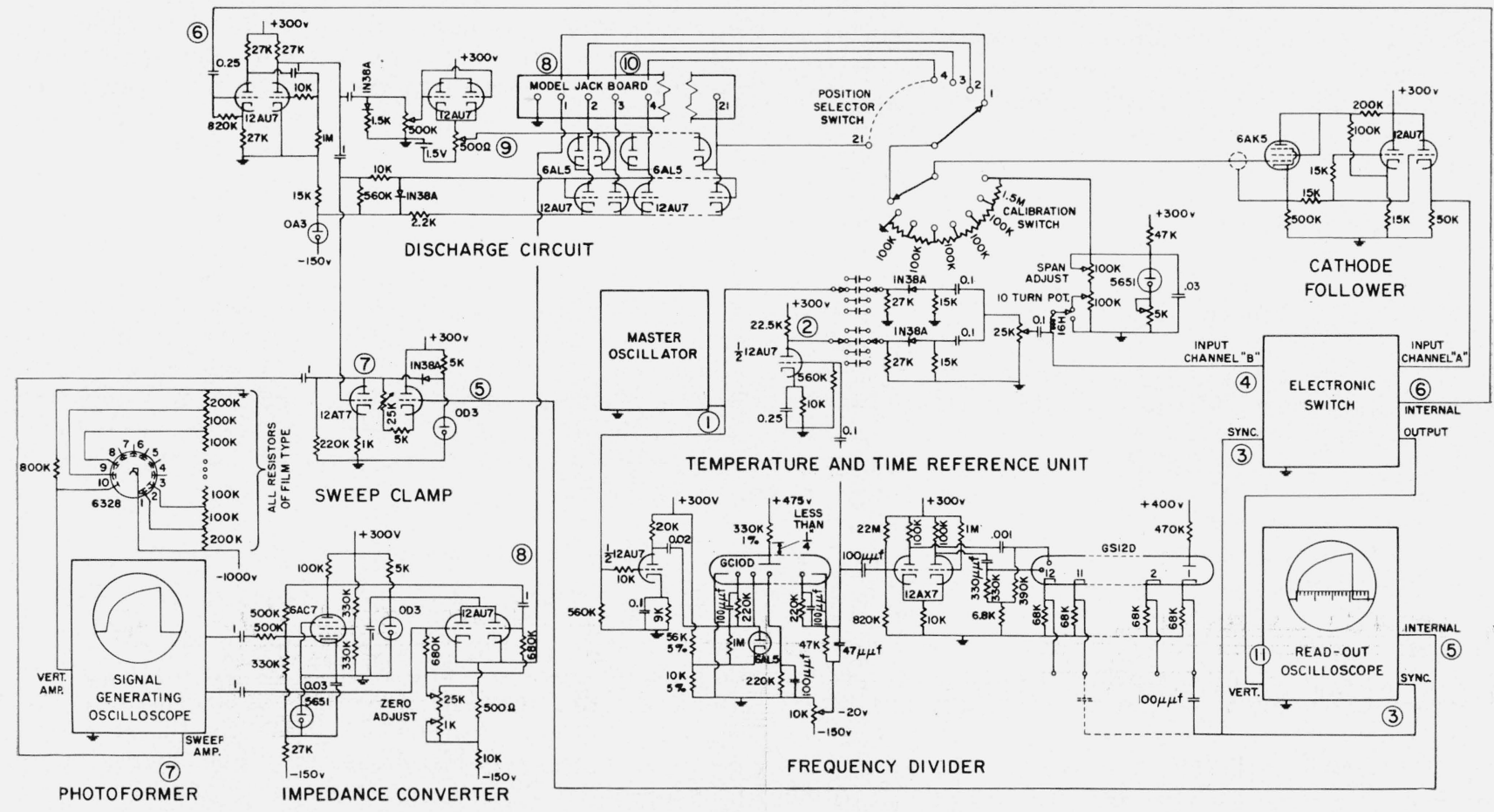

Figure 6. Circuit diagram of analyzer.

Capacitance values are in microfarads unless otherwise indicated in micromicrofarads $(\mu \mu \mathrm{f})$. 


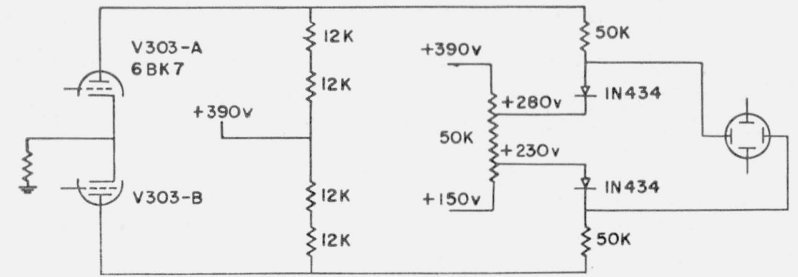

OUTPUT STAGE OF HORIZONTAL HORIZONTAL DEFLECTION DEFLECTION AMPLIFIER

CLAMP CIRCUIT

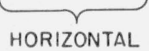

DEFLECTION PLATES

FIGURE 7. Clamp for horizontal deflection circuit of generating oscilloscope.

seemed desirable to assist in preventing "burning" of the phosphor of the cathode-ray tube screen. The oscilloscope used was provided with a beamblanking circuit for removal of the return trace image. This feature was undesirable in the unit as used for signal generation and the instrument controls were accordingly set to remove this circuit from operation.

\subsection{Impedance Converter}

This circuit was designed to combine the balanced but out-of-phase voltages applied to the vertical deflecting plates of the generating oscilloscope into a single-phase output signal with ground as a reference level. Capacity coupling was used, although direct coupling if practical would have provided for more rapid and easy adjustment of the output level with respect to ground. The pentode is used here as a unity-gain phase inverter and the twin triode as an averaging circuit. This was acceptable as a substitute for a true addition circuit because of the very similar size of the two signals being averaged. The output impedance of the converter as measured by the shunt resistance required for 50-percent attenuation of signal was found to be about 250 ohms.

\subsection{Sweep Clamp}

The horizontal timing sweep used for forming the input signal was obtained from an internal connection to the sweep-generating circuit of the viewing oscilloscope. The sweep clamp provided a means for rejecting every alternate sweep cycle and thus only generating an input signal when it was necessary to apply one to the model.

\subsection{Cathode Follower}

This circuit is used as a high-impedance probe feeding a wide-band impedance converter. The circuit while probably not original with us has the unique feature of permitting high-impedance operation with large input signals. The potential divider between plate and cathode of the first stage of the twin triode serves as a variable plate-supply source for the $6 \mathrm{AK} 5$ used as cathode-follower input stage. In this manner large voltage signals can be followed while at the same time limiting the potential drop across the input tube. The input impedance as measured by the decay of voltage on a capacitor was. found to be at least $8,000 \mathrm{meg}$. Frequency response was highly linear up to $25 \mathrm{kc}$. The output of this circuit is mixed with the time and calibration signal by the electronic switch.

\subsection{Master Oscillator}

The time-reference standard for the unit comprises a variable-frequency square-wave oscillator. The output of this unit was fed to both the temperature and time-reference unit, and the frequency divider. The former comprised differentiating, rectifying, and mixing circuits.

\subsection{Frequency Divider}

This circuit made use of "Dekatron" glow-counter tubes of both scale of 10 and scale of 12 types in series. The former permitted accentuation of each tenth timing mark while the latter permitted generation of a synchronizing signal as a submultiple of the scale of 10 count. The output from each of the 12 cathodes of this tube were separately available so that considerable flexibility was possible in generating a time signal appropriate to the input signal being used. The output from this scale of 12 count was used to synchronize the time sweep of the viewing oscilloscope and the switching frequency of the electronic switch. An internal connection was made to the electronic switch to provide a square-wave output in synchronism with the trigger input signal. 'This output was used to operate both the sweep clamp and the discharge circuit.

\subsection{Discharge Circuit}

This unit comprises three circuits: (a) A twin triode amplifier and phase inverter, the output of which is split into two signals to drive; (b) the discharge triodes for each individual section of the model; and (c) the clamp-diode reference-level generator. 'This last circuit was necessary to permit balancing out the potential drop across the clamp diodes and thus to permit discharge of the network model to the ground-reference level. In the original unit, 20 discharge triodes consisting of 10 twin triodes, were used permitting complete discharge of a 20-section electrical model. The discharge circuit was assembled as a unit in a relay-rack section, 3 in. high. A total of 3 discharge circuit assemblies could be installed in the relay rack illustrated in figure 5. This corresponds to a model requiring 60 discharge points.

\subsection{Read-Out or Viewing Oscilloscope}

The same type of oscilloscope was used for observation of behavior of the model as was used for the signal generator. This was done largely because of the facilities it provided for driven-sweep operation from an external trigger signal. This together with the requirement of a highly linear time-sweep source for the signal generator are the only essential requirements of the unit. High linearity of the vertical 


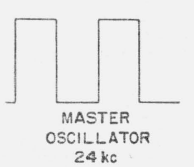

(5)

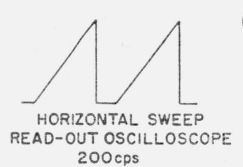

(9)

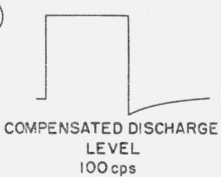

(2)

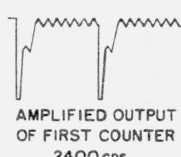

(6)

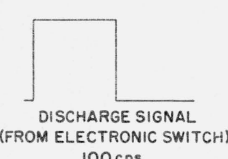

(10)

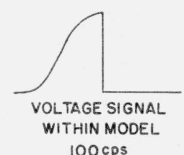

(3)

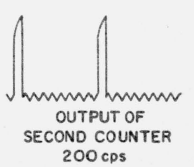

(7)

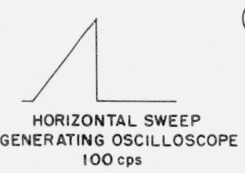

(11)

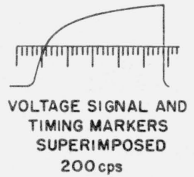

(4)

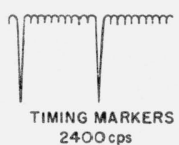

(8)

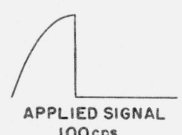

$100 \mathrm{cps}$

FIgure 8. Waveforms experienced in normal circuit operation with master oscillator frequency of $24 \mathrm{kc}$.

Numbers shown correspond to small encircled numbers in circuit diagram.

deflection amplifiers is not essential because of the method used in comparing two different input signals.

Figure 8 presents the waveforms observed with the use of an oscilloscope with 2-meg input impedance. The points on the circuit at which measurements were made with respect to ground are indicated in figure 6 by small encircled numbers. These waveforms have been presented for the case in which the standard time versus temperature curve was being used as the input signal and the master oscillator was set for $24-k c$ operation.

\section{Performance and Limitations}

The performance of the device and the limitations on its use will be described in terms of the errors involved and a comparison between the results obtained by means of analog measurements and those obtained analytically and experimentally. To date, our main interest has been with one-dimensional problems, but the extension to 2 and even 3 dimensions presents practical rather than theoretical difficulties.

\subsection{Errors}

The errors involved in the solution of transient heat-flow problems by means of the electrical analogy described may be divided into (1) errors due to "lumping," also known as truncation errors, and (2) errors due to experimental sources. Since an iterative process is not involved, the errors due to rounding off introduced in numerical computations are absent.

\section{a. Errors Due to Lumping}

These are errors introduced when a line consisting of distributed parameters is represented by one composed of a finite number of sections or lumps. The error is a function of the boundary and initial conditions, the number of lumps, the type of lumping element used (i. e., whether $\mathrm{T}, \mathrm{L}$, or $\mathrm{Pi}$ ), and the ratio of the time at which measurements are taken to the resistance-capacitance product of the electrical model (the Fourier number). Error analyses are so far available for only a very few types of problems. Lawson and McGuire [2] have shown that for a semiinfinite slab, the surface temperature of which was instantaneously raised to and maintained at a constant level, the temperatures for $\mathbf{T}, \mathbf{L}$, and $\mathrm{Pi}$ section networks are identical after the first section. Klein, Touloukian, and Eaton [11] have analyzed the distribution of errors in a finite bar (represented by different numbers of $\mathbf{T}$-sections) one end of which is instantaneously raised to a temperature that is maintained constant thereafter while the other end is maintained at a constant reference temperature. They found that departure of the lumped system from the distributed system at the center of the bar was reduced (after high positive departures during the very early part of the transient) from a maximum of -6 percent with a 5 -section model to a maximum of -2 percent with an 11-section model. Greater departures were found toward the leading section in a lumped system so that this point may yield unreliable information for the earliest part of the transient. Clarke [12] has analyzed the error in a finite bar represented by different numbers of $\mathbf{L}$ sections, one end of which is supplied with a constant heat flux, the other end being perfectly insulated. He found that departure of the lumped system from the distributed system at the insulated end of the bar was always positive and decreased with the time at which measurements are made. Friedmann [13] has estimated an upper bound for the global or maximum error between the exact solution of the heatconduction equation examined by Klein et al. and the exact solution of its lumped parameter analog.

The numerical solution for the distribution of lumping errors in the problem of a finite bar with perfectly insulated sides, one end of which is instantaneously raised to a temperature that is maintained constant thereafter while the other end remains perfectly insulated, seems to have been overlooked by workers in this field. The solution for the lumped system of $\mathbf{T}$-sections was found in Jaeger [14], while the exact thermal solution was given by Carslaw and Jaeger [15]. Some of the results of the analytical calculations are shown in a series of curves in figures 9 and 10 . The comparison between the errors at the center in each of the lumped circuits as shown in figure 9 indicates the improvement in the results as more sections are used to represent the distributed system. For a given lumped circuit of 10 sections, error curves for three points (corresponding to the lengths $0.05,0.25$, and 0.75 ) are shown in figure 10 . The errors are greatest at the first section and attenuate rapidly for points further removed from the 
source. A similar series of curves were derived from the data of Klein et al. for an 11-section system and are shown in figure 11. The similarity in the error distribution for the cases of short- and open-circuit terminations suggests that changes in the circuit termination do not result in appreciable changes in the distribution of errors within a lumped line.

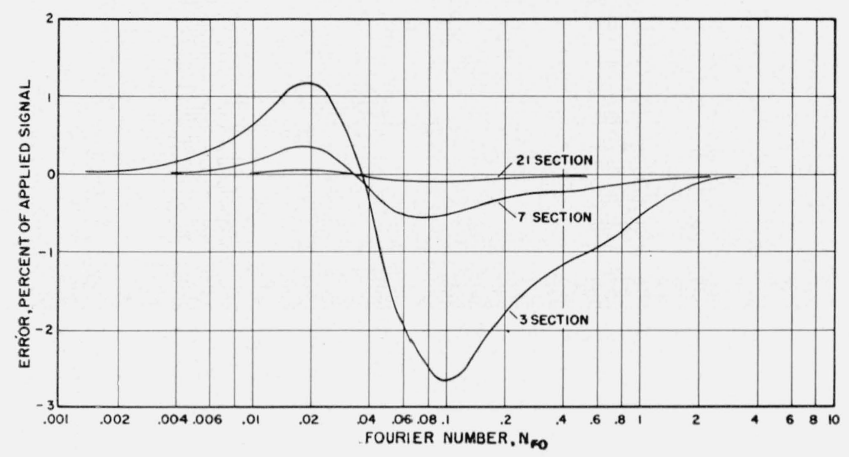

FIGURE 9. Lumping error at the center of a system represented by 3,7 , and 21 T sections.

Open-circuit termination.

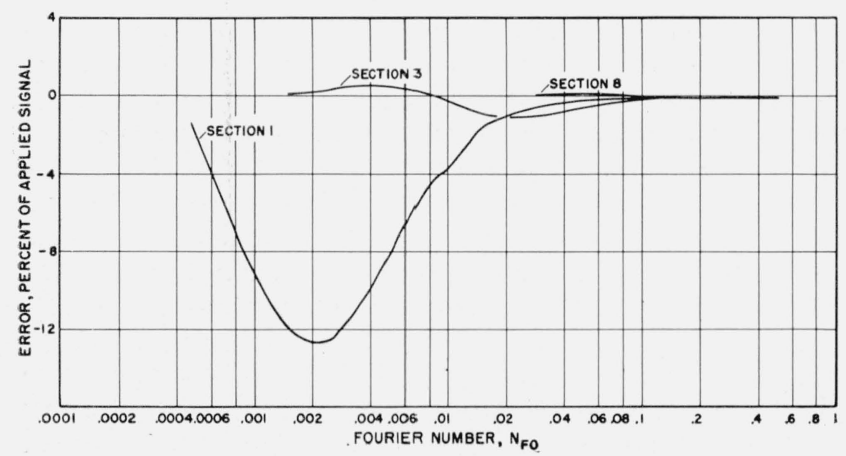

FIGURE 10. Lumping error at sections 1, 3, and 8 in a system represented by $10 \mathbf{T}$ sections.

Open-circuit termination.

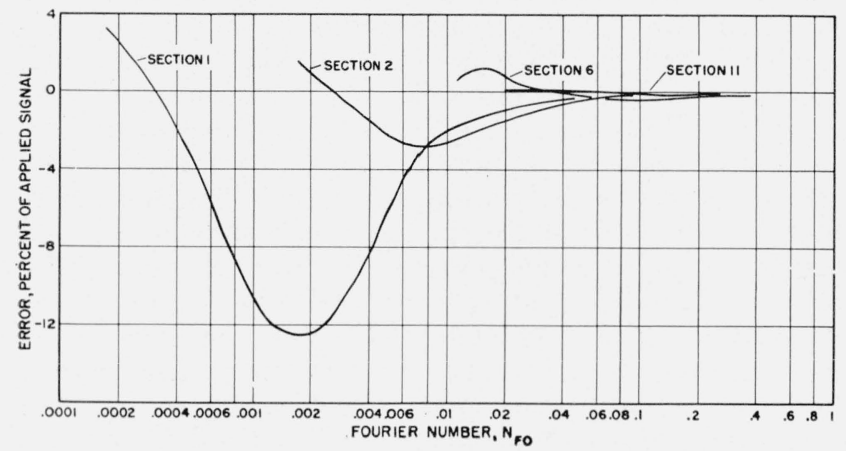

Figure 11. Lumping error at sections 1, 2, 6, and 11 in a system represented by $11 \mathbf{T}$ sections.

Short-circuit termination. Data from reference [11].

\section{b. Errors Due to Experimental Sources}

These include (a) consistent errors due to the measuring-circuit impedance and response, or due to leakage and stray currents arising from the network capacitors or the mounting system; and (b) random errors in measuring capacitance, resistance, voltage, time, etc. Paschkis and Heisler [16] have shown that for the case where leakage is significant, the error due to network capacitor or mounting system leakage decreases as the number of sections of the network decreases. The versatility of the time scale employed here permits operation with network resistors below the megohm range and capacitors below the microfarad range for all materials under study, thereby minimizing stray currents due to the network elements. The parallel impedance introduced by the discharge-circuit elements and the measuring circuit is of the order of several thousand megohms. The extent to which the photoformer can generate a square-wave pulse at the operating frequencies is limited to about 1 percent of the time period available for measurements. The over-all sweep and signal-generation linearity of the device as measured with a linear-rise input function was found to be within the sweep-linearity specification of the oscilloscope alone, namely, "any 10-percent increment is within 10 percent of any other 10-percent increment ..." It is estimated that for the onedimensional case errors due to all experimental sources (which limit the accuracy of the method) are within 2 percent of full-scale. This is entirely apart from the much larger uncertainty associated with the values of the thermal properties of materials.

\subsection{Comparison With Analytic Solution}

Figure 12 is a comparison between the analog and analytic solutions for temperature curves within a

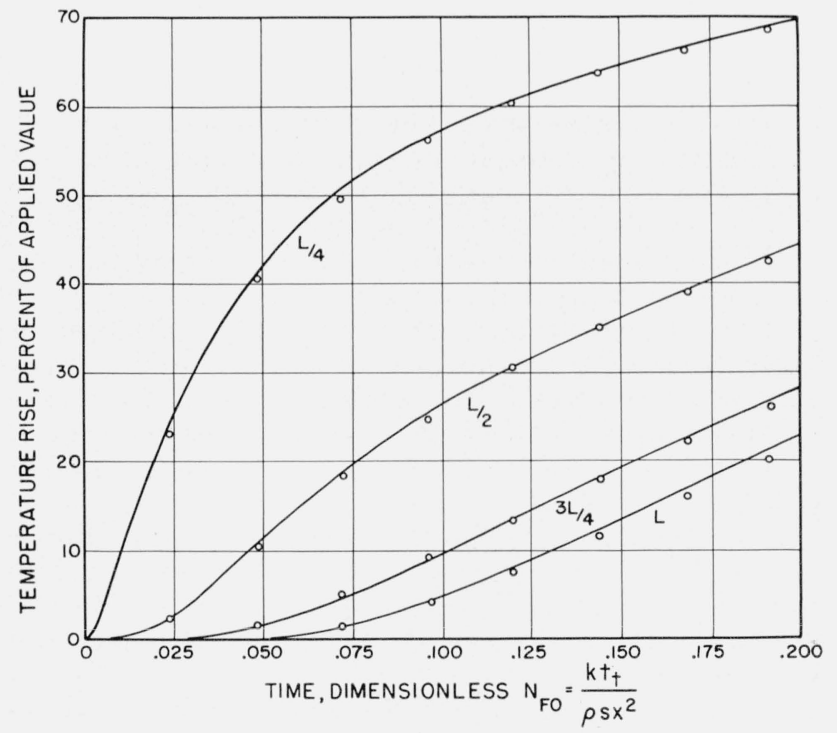

FIGURE 12. Comparison between analog and analytic solutions for a one-dimensional problem.

_- Analytic solution; $\bigcirc$, analog solution. 
finite bar with perfectly insulated sides, one end of which is instantaneously raised to a temperature that is maintained constant thereafter while the other end remains perfectly insulated. From the preceding discussion on errors and since we are using a 20section network representation, it is evident that the difference between the analog and analytic solutions are principally due to experimental sources. The maximum error is seen to be within the estimated 2 percent of the applied signal.

\subsection{Comparison With Experimental Results}

Figure 13 is a comparison between the analog and experimental results on a horizontal 4 -in. concrete slab the lower surface of which was subjected to the

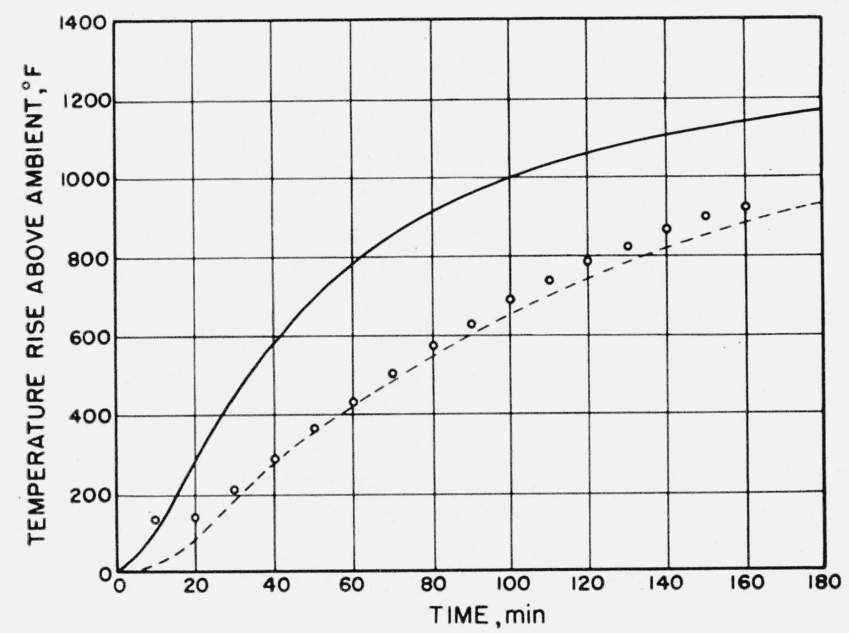

FIGURE 13. Temperature rise at center-plane of a 4-in. concrete slab subjected to standard fire exposure.

, Analog solution $s=0.837$ joule $/ \mathrm{g}{ }^{\circ} \mathrm{C} \quad \ldots$..., analog solution $s=2.09$ joules $/ \mathrm{g}$ ${ }^{\circ} \mathrm{C} ; \mathrm{O}$, experimental results.

standard fire exposure. This problem was set up as an illustrative example in section 2 and figure 14 shows the form of read-out oscilloscope display for such a problem. In normal use only two curves would be displayed simultaneously; the calibration scale, and a temperature versus time curve for the position under study. However, for purposes of illustrating a larger portion of the solution, timetemperature curves for a number of locations within the specimen as well as the two surfaces have been recorded by means of multiple photographic exposures.

Convective and radiative heat loss on the upper surface has been represented by the appropriate varistor assembly. The analog result was obtained using constant values of density, specific heat, and thermal conductivity as noted in figure 2. The device is not set up to handle temperature or timevarying properties although these variations over the temperature range considered may be appreciable. Since the values themselves are subject to

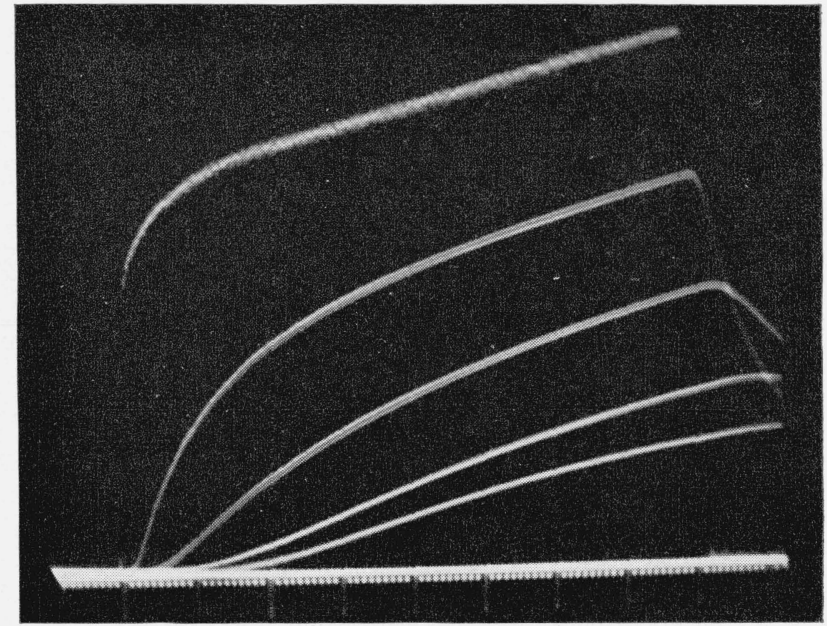

FIGURE 14. Multiple-exposure photograph of read-out oscilloscope display for transient one-dimensional heat flow in a homogeneous slab.

Large time-scale markers correspond to 1-hr time periods.

considerable uncertainty, their variation with temperature may be considered only a refinement. However, the effect of variable properties may be quickly investigated by duplicate tests at several different but constant values of the property in question. Or, alternately, by designing a line such that the thermal constants represented at each section vary as the mean temperature likely to be obtained at those points. The broken line in figure 13 represents the temperature curve for a specfic heat of 2.09 joules $/ \mathrm{g}{ }^{\circ} \mathrm{C}\left(0.5 \mathrm{Btu} / \mathrm{lb}^{\circ} \mathrm{F}\right)$ and illustrates how the device may be used to estimate thermal properties when direct thermal property measurement is impractical. The extent of the physical changes taking place during fire exposure and the role that moisture plays are a few of the uncertain variables which may cause considerable difference between experimental and analog results.

\section{Conclusions}

An electronic instrument has been constructed which permits the solution of transient heat-flow problems by means of analogous resistance-capacitance electrical networks. On the basis of the work completed, the reliability of the instrument as well as its flexibility with regard to input-signal waveform and time scale have been demonstrated. For an assumed one-dimensional problem in which thermal properties were independent of temperature and high accuracy was achieved in the construction of the electrical model, the errors were found to be within 2 percent of the applied signal. The instrument has been applied to a variety of problems in the field of fire research where a rapid estimation of the thermal endurance of structures of given thermal properties may be obtained. Although not yet attempted, the method may be extended to two- and three-dimensional transient heat-flow problems. 
The authors acknowledge the assistance of Dwight Y. Sumida for performing the computations necessary for the error analysis, of James S. Evans, Jr., now with Hughes Aircraft Company, for design of the cathode-follower circuit, and of William H. Bailey for aid in assembling the device and for the preparation of the illustrations. They also appreciate the kind permission granted by Mr. E. O. P. Klein and by Professors Y. S. Touloukian and J. R. Eaton of Purdue University to print the results shown in figure 11 .

\section{References}

[1] American Society for Testing Materials, Standard methods of fire tests of building construction and materials, ASTM Designation E110-55.

[2] D. I. Lawson and J. H. McGuire, The solution of transient heat flow problems by analogous electrical networks, Inst. Mech. Engr. Proc. [A] 167, 275 (1952).

[3] G. Kirchhoff, Flow of an electric current through a plane, particularly through a circular dise (in German) Ann. Phys. Chem. 64, 499 (1845); 67, (1846).

[4] T. J. Higgins, Electroanalogic methods, Appl. Mechanics Rev., p. 1 (Jan. 1956); p. 49 (Feb. 1956); and p. 49 (Feb, 1957)

[5] W. W. Soroka, Experimental aids in engineering design analysis, Mech. Eng. 71, 831 (Oct. 1940).

[6] W. W. Soroka, Analog methods in computation and simulation (McGraw-Hill Book Co., Inc., New York, N. Y., 1954).

[7] L. Beuken, Construction of an electrical analogy apparatus for the analysis of non-stationary heat flow, Econ. Tech. Tijdschr. 19, No. 3, 43 (June 1939).

[8] V. Paschkis and H. D. Baker, A method for determining unsteady state heat transfer by means of an electrical analogue, ASME Trans. 64, 105 (Feb. 1942).

[9] D. M. MacKay, A high-speed electronic function generator, Letter to the Editor, Nature 159, 406 (March 22,1947 )

[10] D. J. Mynall, Electronic analog computing, Electronic Eng. 19, 259 (1947).

[11] E. O. P. Klein, Y. S. Touloukian, and J. R. Eaton, Purdue University (personal communication).
[12] L. N. Clarke, The effect of the number of sections on the accuracy of a particular RC electrical analogue, Aus. tralian J. Appl. Sci. 3, No. 2, 119 (June 1952).

[13] N. E. Friedmann, Truncation error in a semi-discrete analogy of the heat equation, J. Math. Phys. 35, No. 3, 299 (Oct. 1956).

[14] J. C. Jaeger, Introduction to the Laplace transformation, p. 52 (Council for Scientific and Industrial Research, Melbourne, Australia, Jan. 1946).

[15] H. S. Carslaw and J. C. Jaeger, Conduction of heat in solids, p. 83 (Oxford University Press, 1947).

[16] V. Paschkis and M. P. Heisler, The accuracy of measurement in lumped $\mathrm{R}-\mathrm{C}$ cable circuits as used in the study of transient heat flow, Trans. AIEE 63, 165 (1944).

\section{Appendix. Instrument and Components List}

The special components and instruments used in the assembly are listed below. It is quite possible that other equally satisfactory components could have been used.

Generating oscilloscope. DuMont type 340-R, cathode ray tube type 5AQP-15, short-persistence phosphor.

Viewing oscilloscope. DuMont type $340-\mathrm{R}$, eathode ray tube type $5 \mathrm{AQP}-1$.

Oscillator. Rutherford Electronics Co., model G1 pulse and square wave oscillator. Frequency range 1 to 100,000 pulses per second.

Electronic switch. DuMont type 330, arranged for triggered operation

Multiplier phototube. RCA-6328, 9-stage type with S-4 response.

Photomultiplier power supply. C. J. Applegate model 123A, $1,000 \mathrm{v}$ "Uniplug".

Counter tubes. Distributed by Atomic Instrument Co. One type GC10D single decade (scale of 10) and one type GS12D (scale of 12) "Dekatron" cold cathode glow transfer counter tubes.

Direct-current voltage supplies. Low-voltage regulated supply, $-150 \mathrm{v}, 125 \mathrm{ma}$. High-voltage regulated supply, $+300 \mathrm{v}$, $300 \mathrm{ma}$.

Washington, April 29, 1958. 